\section{REDUCE THE RISK OF DAMAGING A RESTORATION}

The Enhance Composite Finishing and Polishing System has a one-piece disc and mandrel and can significantly reduce the risk of damaging a restoration. Enhance should be used in slow speed handpieces with speeds ranging from 10,000 rpm to 15,000 rpm.

The aggressiveness of the Enhance disc is controlled by the pressure applied to the surface of the restoration. The greater the pressure, the more material is removed. Lighter pressure leaves a smooth surface.

The polishing sequence is completed by using Enhance polishing cups and Prisma-Gloss Regular and Extrafine

\section{ACCURATE COLOUR EVALUATION}

Accolade PV Veneer Placement System from Danville is available in the UK from Evident. Accolade PV includes a unique try-in paste which is placed directly on the silane, allowing the veneer to be simultaneously tried-in for fit and colour. Accolade PV's tryin paste and bonding composite are the same material, however the try-in paste has no light sensitive initiators, allowing accurate colour evaluation

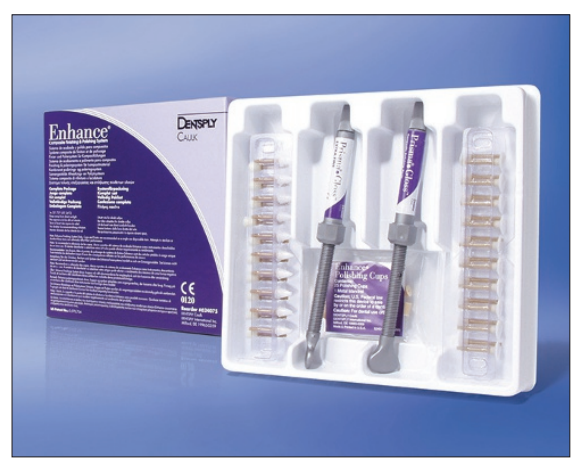

pastes. Enhance, available as single-use items in three shapes, discs, points and cups, can finish and pre-polish all types of resin-based restoratives. The complete system of Enhance enables you to achieve microfine-like polish.

Reader response number 63

with nearly unlimited try-in time.

Using a composite rather than a glycerine-based try-in material not only speeds up the procedure but also eliminates the possibility of contamination by the try-in material. After try-in, the dentist removes at least 50\% of the Accolade PV try-in paste and replaces it with the normal catalysed Accolade PV composite. The remnant try-in material is polymerised by diffusion polymerisation.

Reader response number 64

\section{UNIQUE PARTICLE STRUCTURE}

GC Gradia Direct features shade adaptation to the surrounding tooth structure, and provides you with aesthetic results due to the unique particle structure and light reflectiveness.

When dealing with more demanding and complex cases a combination of different shades enables unlimited aesthetic

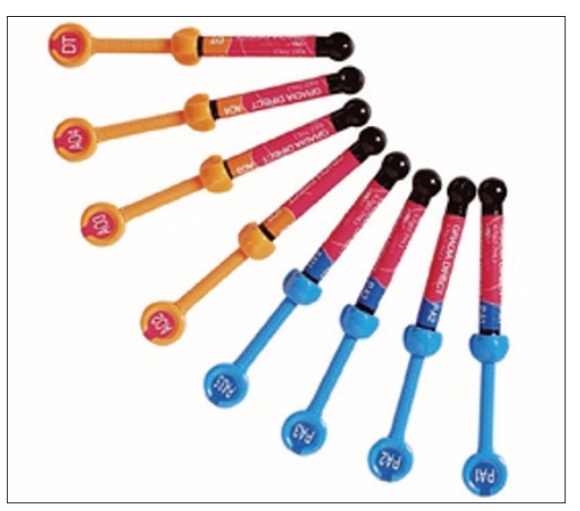

results. GC Gradia Direct offers you the complete assortment from bleach white to dark translucent to outside special shades.

Radiopaque GC Gradia Direct Posterior combines aesthetics with mechanical properties by means of high fracture toughness to resist occlusal stress, high wear resistance and low wear on opposing dentition.

GC have developed a new shade guide that takes into account the chroma, hue and value of each composite shade whether you have opted for a single shade or if you are extensively restoring a tooth and require multiple shades.

This shade guide incorporates two special shades, which are placed underneath or on top of a standard shade to further enhance your restoration.

Reader response number 65

\section{OPTIONS FOR BUILDING BONE}

BIOMET $3 i$ has expanded its regenerative portfolio to offer a variety of options for building bone. Endobon Xenograft Granules are bovine derived hydroxyapatite that enables bone to grow directly on the ceramic surface and through the entire graft. The mineralised nature of Endobon Xenograft Granules provides a nonresorbable osseoconductive scaffold that is ideally suited for regeneration of defects when effective space maintenance is required.

Endobon Xenograft Granules are fully deproteinated by a two-step high temperature manufacturing process for safety from bacteria, viruses and prions and have excellent handling characteristics for easy transfer to the defect site. Endobon Xenograft Granules with an Osseoguard Membrane complete the regenerative product portfolio of BIOMET $3 i$.

Reader response number 66

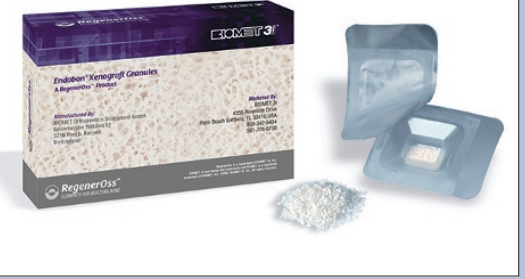

\section{SHORT CURING TIME}

The SmartLite PS is a curing light which is small, silent, lightweight and completely cordless. It offers high performance with short curing times, and is available in three colours with no difference in technical specifications original dark blue version, sunset version and blue sky version.

Reader response number 67

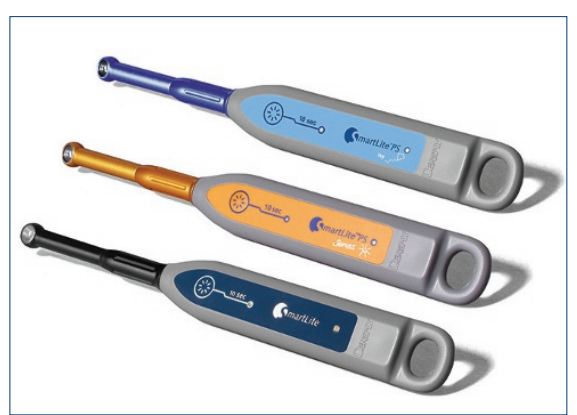

